Forum

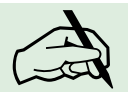

\section{Fortbildung gemäss FBO}

Heute wende ich mich mit einem Problem bezüglich Fortbildung an Sie, welches sowohl mich als auch mehrere meiner ärztlichen Kollegen betrifft.

Ich bin zu 100\% angestellt im Medizinischen Dienst der IV-Stelle des Kantons Zürich in beratender ärztlicher Funktion. Gemäss der FB0 vom 24. Juni 1998 (ab 1. Januar 2000 gültig für alle Mitglieder der FMH) bin ich zu 80 Stunden Fortbildung (maximal 30 Stunden Selbststudium) verpflichtet. Eine Basisfortbildung von mindestens 40 Stunden jährlich ist möglich. Nun habe ich Probleme, diese Pflicht zur Fortbildung umzusetzen derart, dass mein Vorgesetzter (Jurist) Fortbildungen nur zulässt, wenn sie unmittelbare Relevanz für den Betrieb der Invalidenversicherung haben (und das auch nur nach vielerlei Diskussionen; über die Relevanz entscheidet er selbst). Im besonderen wurde eine Fortbildung für Grundversorger über fünf Tage in Davos abgelehnt, welche ja zur Auffrischung und Aktualisierung des allgemeinen medizinischen Wissens und der Erfüllung der Fortbildungspflicht gedient hätte. Nun steht in Art. 2 der FBO, dass die Ärzteschaft Umfang und Inhalt der Fortbildung bestimmt. Nun zu meinen Fragen:

1. Inwiefern muss der Arbeitgeber mich unterstützen, meiner Fortbildungspflicht nachzukommen?

2. Kann er von mir verlangen, dass ich Fortbildungen, die ganz klar dem Art. 1 der FBO entsprechen, er aber für nicht relevant erachtet, in meiner Freizeit absolvieren (Urlaub, Überzeit, etc.)?
3. Muss er mich für derartige Fortbildungen unter Berücksichtigung betriebsinterner Aspekte wie Ferienabsenzen anderer etc. freistellen (bezahlter Urlaub)?

4. Oder bestimmt ausschliesslich der Arbeitgeber über Nutzen oder Nichtnutzen einer Fortbildung und kann demnach selbst frei entscheiden, welchen Anteil er an den Fehltagen und den Kosten trägt?

Vielen Dank für die Bearbeitung der Fragen und freundliche Grüsse

\section{Dr. med. Frank Peric, Langwiesen}

\section{Replik}

Die von Ihnen geschilderte Problematik ist uns bereits von verschiedener Seite herangetragen worden. Vor allem Oberärzte werden oft nur unzureichend für die adäquate Benutzung von Fortbildungsangeboten freigestellt bzw. finanziell unterstützt.

Die in der FBO der FMH stipulierten zehn Tage Fortbildung stellen ein Minimum dar, um die einmal erworbene ärztliche Kompetenz zu erhalten und aufgrund der Entwicklungen der Medizin zu aktualisieren. Dies gilt selbstredend nicht nur für praktizierende Ärzte, sondern auch für ärztlich tätige Angestellte in der Verwaltung und in der Industrie. Unter diesem Gesichtspunkt scheint es mir selbstverständlich zu sein, dass der Arbeitgeber Fortbildungsbemühungen von angestellten Ärzten weitgehend unterstützt. Dabei ist der Besuch von Fortbildungsveranstaltungen, welche mit betrieblichen Notwendigkeiten nicht kollidieren, wohl unbestritten. Rechtlich heikel wird es bei der Frage, ob die dafür aufgewendete Zeit als Arbeitszeit zu gelten hat. Massgebend sind hier die anwendbaren arbeitsrechtlichen Bestimmungen (in Ihrem Fall wahrscheinlich das Personalrecht des Kantons Zürich und der Arbeitsvertrag).
Falls und soweit das allgemeine Obligationenrecht anwendbar ist, sind obligatorische Fortbildungskurse analog zu Krankheit etc. als bezahlte Abwesenheiten zu betrachten. Das Freizügigkeitsgesetz, das die Weiterund Fortbildung der Medizinalpersonen bundesrechtlich regelt und voraussichtlich im Jahr 2001 in Kraft treten wird, sieht ausdrücklich ein Fortbildungsobligatorium vor. Vor diesem Hintergrund ist es vertretbar, wenigstens einen Teil Ihrer Fortbildungsbemühungen als Arbeitszeit gelten zu lassen. Kaum zu beanstanden ist, wenn der Arbeitgeber hauptsächlich diejenige Fortbildung unterstützt, die Sie für Ihre konkrete berufliche Tätigkeit benötigen. Da der Arbeitgeber für Ihre Tätigkeit vermutungsweise den FMHTitel voraussetzt, sollte er auch weitere, im anwendbaren Fortbildungsprogramm vorgesehene Veranstaltungen berücksichtigen. Ich hoffe, Ihnen mit diesen Angaben zu dienen und stehe Ihnen für allfällige Rückfragen gerne zur Verfügung.

Mit freundlichen Grüssen

Ch. Hänggeli, Abteilung Weiter- und Fortbildung

\section{Anmerkung}

Das Problem hat sich inzwischen wie folgt gelöst:

Sämtliche Ärzte der IV-Stelle Zürich werden ab sofort für bis zu zehn Fortbildungstage jährlich gemäss ihrer eigenen Wahl freigestellt, wobei die IV-Stelle Zürich die üblichen Kosten gemäss Spesenreglement übernimmt (Kurskosten, Unterkunft, Verpflegung, Reisekosten). Sozusagen als Gegenleistung erstatten die Kollegen einen Bericht über den Inhalt der Veranstaltung im Rahmen einer internen Kurzfortbildung.

\title{
Personalien / Nouvelles du corps médical / Dati personali
}

Todesfälle

\section{Décès}

Decessi

Jean-Paul Gonseth (1921)

Facharzt FMH für Psychiatrie und Psychotherapie, 4410 Liestal

\section{Ehrungen}

\section{Distinctions}

The $5^{\text {th }}$ Congress of the International Xenotransplantation Association and Novartis Pharma congratulates L. Bühler for his presentation "A nonmyeloablative regimen with CD40L blockade leads to humoral/cellular hyporesponsiveness to pig hematopoietic cells in baboons" and is happy to support this study with a Novartis Award.

Prof. Dr. med. Dr. h.c. Jürg Willi-Dubach, Zürich, wurde anlässlich des Dies academicus der Universität Fribourg auf Antrag der Faculté de sciences zum Ehrendoktor Dr. rer. nat. h.c. ernannt.

\section{Ärztegesellschaft des Kantons Bern}

\section{Medizinischer Bezirksverein Bern-Stadt}

Zur Aufnahme hat sich angemeldet:

als ordentliches praktizierendes Mitglied: Dr. med. Christine Gerber Rihs, Fachärztin FMH für Allgemeinmedizin, Praxis Bubenberg, Bubenbergplatz 11, 3011 Bern

Einsprachen gegen diese Kandidatur müssen innerhalb von 14 Tagen seit dieser Veröffentlichung schriftlich und begründet dem Präsidenten des Medizinischen Bezirksvereins Bern-Stadt eingereicht werden. Nach Ablauf der Einsprachefrist entscheidet der Vorstand über das Gesuch und allfällige Einsprachen.

Der Vorstand des MBV Bern-Stadt 